\title{
The Effect of Compression on the Void Coalescence under Strong Dynamic Loading
}

\author{
Ruichen Tu, ${ }^{1}$ Ning Wei $\left(D,{ }^{1}\right.$ Yongmao Pei, ${ }^{2}$ Yan Liu, ${ }^{3}$ Fengguo Zhang, \\ and Dongsheng Zhang ${ }^{1}$ \\ ${ }^{1}$ School of Mechanical Engineering, Shaanxi University of Technology, Hanzhong 723000, China \\ ${ }^{2}$ College of Engineering, Peking University, Beijing 100871, China \\ ${ }^{3}$ School of Aerospace Engineering, Tsinghua University, Beijing 100084, China \\ ${ }^{4}$ Institute of Applied Physics and Computational Mathematics, Beijing 100094, China \\ Correspondence should be addressed to Ning Wei; nwxyh0606@163.com
}

Received 16 March 2021; Accepted 11 January 2022; Published 28 February 2022

Academic Editor: Daniela Pilone

Copyright ( $\odot 2022$ Ruichen Tu et al. This is an open access article distributed under the Creative Commons Attribution License, which permits unrestricted use, distribution, and reproduction in any medium, provided the original work is properly cited.

The void coalescence under strong dynamic loading is a significant spallation process for ductile metals. Since the spallation is basically dominated by tension waves, most void coalescence studies have focused on the tension effect. However, it is known that in spallation, the material initially undergoes a strong compression wave, and then an irreversible deformation is produced by the compression wave inside the material. Therefore, in this paper, the effect of compression on the void coalescence is investigated using the molecular dynamics (MD) simulation. It was found that as the compressive strain increases, the yield strength decreases first and then increases. The results showed that due to the Bauschinger effect (BE), the yield strength decreases by $19.43 \%$ from $5.66 \mathrm{GPa}$ without compressive loading to $4.56 \mathrm{GPa}$ when the compressive strain is $-7.5 \%$, after which the yield strength increases. The voids do not coalesce when the compressive strain is $-8 \%$. In addition, it was found that during the compressive phase, the void surfaces would generate dislocations, which could obstruct the void coalescence in the tensile phase. Furthermore, under compressive loading, the temperature effect on the void coalescence was studied, and it was found that lower temperatures could suppress the void coalescence.

\section{Introduction}

Dynamic tensile fracture (spallation) of ductile metals under strong dynamic loading is a typical failure mode of material dynamic damage, which is also a fundamental problem in engineering. Under strong dynamic loading, the shock wave interaction in opposite directions generates a stress pulse in ductile metals, which induces microvoid nucleation, growth, and coalescence corresponding to the accumulation, growth, and rapid development of damage. Once the damage has accumulated to a certain extent, it causes material failures. As the rapid growth of damage is a critical process which causes material failure, the mechanism of the void coalescence needs to be further studied.

The relations between the void coalescence and void sizes, the mutual position of voids, loading conditions, and material properties have been studied in many references.
Tvergaard et al. [1,2] introduced two adjustment parameters to consider the interaction between voids. A model, which is known as the Gurson-Tvergaard-Needleman (GTN) model $[3,4]$, has taken into account the process of rapid loss of load carrying capacity caused by the void coalescence. Gologanu et al. $[5,6]$ developed a model named Gologanu-Leblond-Devaux (GLD), which considered the effect of void shape evolutions on the void coalescence. Earlier studies often focused on the void coalescence under quasistatic loading, which led researchers to study the case under strong dynamic loading. In order to study dynamic tensile fracture, the split Hopkinson pressure bar (SHPB) loading technology [7] has been widely used for problems with a strain rate of less than $10^{4} \mathrm{~s}^{-1}$ and the laser loading technology [8] for higher strain rate conditions between $10^{5}-10^{7} \mathrm{~s}^{-1}$. A model was established by Thomason et al. [9], and they found that the effects of inertia and strain rate 
influenced the growth of dynamic damage. The results of this model were in good agreement with the relevant experimental results. Moreover, temperature has been found as an important parameter. For instance, the effect of temperature on void evolutions has been confirmed [9-11]; it significantly influenced the critical stress and damage growth rate, and the fracture strength had a negative correlation with the initial temperature. In addition, it is worth mentioning that the distance between two voids and the void radius [12] can also greatly affect the coalescence efficiency. A study [13] found that there was a relationship between the intervoid ligament distance (ILD) of two same voids. When the ILD was equal to the void radius, the voids were most likely to coalesce.

Due to experimental and observational limits, the internal evolution of void coalescence in extreme conditions has not been universally investigated. Therefore, numerical simulation methods are used to study the void coalescence under strong dynamic loading. Jacques et al. [14] revealed the performance difference of samples under quasistatic and strong dynamic loading. The inertia effect of ductile metals subjected to dynamic loads was also studied [15], indicating that this effect on the growth and coalescence of voids is significant. Lubarda et al. [16] pointed out that at higher strain rates, the damage could only grow through surface emission dislocations, and the critical stress of the dislocation gradually decreased with increasing damage size. Horstemeyer et al. [17] proposed a critical ILD to define the distance between two voids, and they employed the micromechanical finite element method (FEM) to quantify the coalescence effect based on temperature and voids that had different spatial arrangements. It is known that the molecular dynamics (MD) method can properly simulate the processes of void nucleation, growth, and coalescence, so that it has gradually become popular for investigating void coalescence. Potirniche et al. [18] proved that the void size and strain rate of loading influence the void growth and coalescence of single crystal nickel. Tang et al. [19] by comparing the metals of face-centered cubic (FCC) crystal with hexagonal close-packed (HCP) crystal, found that the void coalescence mode of the HCP crystal largely depended on crystal orientations. Cui et al. [20] considered the void shape in coalescence and confirmed that the elliptical voids with an oblate-oblate combination would be easier to coalesce. Deng et al. [21] studied the effect of different loading directions on the void coalescence based on the [100] crystal orientation. The results illustrated that the voids were most likely to coalesce when the loading direction was at an angle of $60^{\circ}$ to the centerline of the two voids. Zhang et al. [22] suggested that even if the dislocation of the twin crystal was not as slow as that of a single crystal, the coalescence velocity of the voids of a twin crystal is still faster than that of a single crystal. The twin boundary can reduce the critical stress and suppress dislocations. Peng et al. [23] performed tens of millions of atoms of MD simulations, in which the voids collapsed under an impact loading, while after a tensile loading was applied, the voids were re-initiated during the contraction. Diwu et al. [24] studied the compression of porous voids under high strain rates and revealed that the porous structure under compressive loading would cause the elastic modulus and rheological peak stress of FCC metals to decrease. Liu et al. [25] investigated the effects of void radius and ILD on the void coalescence, and it was found that the rapid growth stage for the void growth was dependent on the initial void radius, and smaller radii can lead to higher growth rates. The first peak value of stress triaxiality increases first and then decreases with the increase of ILD.

However, in spallation, the compression wave is transformed into a tensile wave on the free surface, where it encounters the incident unloading wave and a local tensile stress region is generated. Consequently, the generation of shock waves is often accompanied by compressive and tensile phases. Note that the void coalescence studied using the compressive-tensile loading method is rarely found in the reference. Therefore, in this study, the MD method has been utilized to simulate single-crystal aluminum under uniaxial compressive-tensile plastic deformation in the presence of nanovoids along the [100] crystal orientation. In addition, the effects of temperature and strain rate are also considered in studying the influence of compression on the void coalescence. In this regard, the yield strength of specimens under different compressive strains is also compared.

\section{Simulation Method}

In this study, the large-scale atomic/molecular massively parallel simulator (LAMMPS) was used in the MD simulation [26]. Since the accuracy of the interaction potential function between molecules or atoms has a tremendous influence on the MD method, it is extremely important to choose a suitable potential function. In this simulation, the interatomic interactions of aluminum were described by the embedded atom method (EAM) potential [27, 28].

First, the atomic models for single-crystal aluminum were constructed. In order to eliminate the influence of box size as much as possible, some tensile simulations of the model without voids were set at first. The stress-strain curves of these simulations are shown in Figure 1. When the model size is diminutive, the stress-strain curve oscillates severely, while this curve tends to be stabilized when the size is gradually increased to $30 a$, in which " $a$ " is a unit cell. The value of " $a$ " equals the lattice constant of aluminum metal at $300 \mathrm{k}$; thus, $a=0.405 \mathrm{~nm}$. Considering the stability and capacity of calculations, the side length of the simulated box was set to " $50 a$ ". The dimensions of the specimens were $L=h=50 a$ and thickness $t=25 a$. Two spherical voids were prefabricated in the constructed model with a void radius of $r=5 a$, as shown in Figure 2, and the distance between the two voids' centers was three times the radius [13]. All MD simulations were first relaxed at temperature $300 \mathrm{k}$ and $0 \mathrm{~Pa}$ pressure in three directions for 20 picosecond (ps) using the Nosé-Hoover thermostat and barostat [29]. The periodic boundary conditions were set in all simulated directions. The time step was 1 femtosecond ( $\mathrm{fs}$ ) in all simulations. The loading strain rate was controlled to achieve uniaxial compressive-tensile loading (Figure 2), which was utilized throughout the simulation, and the initial compressive strain was set to eleven groups: $0,-1,-2,-3,-4,-5$, $-6,-6.5,-7,-7.5$, and $-8 \%$. The model was subjected to strain rate controlled uniaxial compressive loading and then 


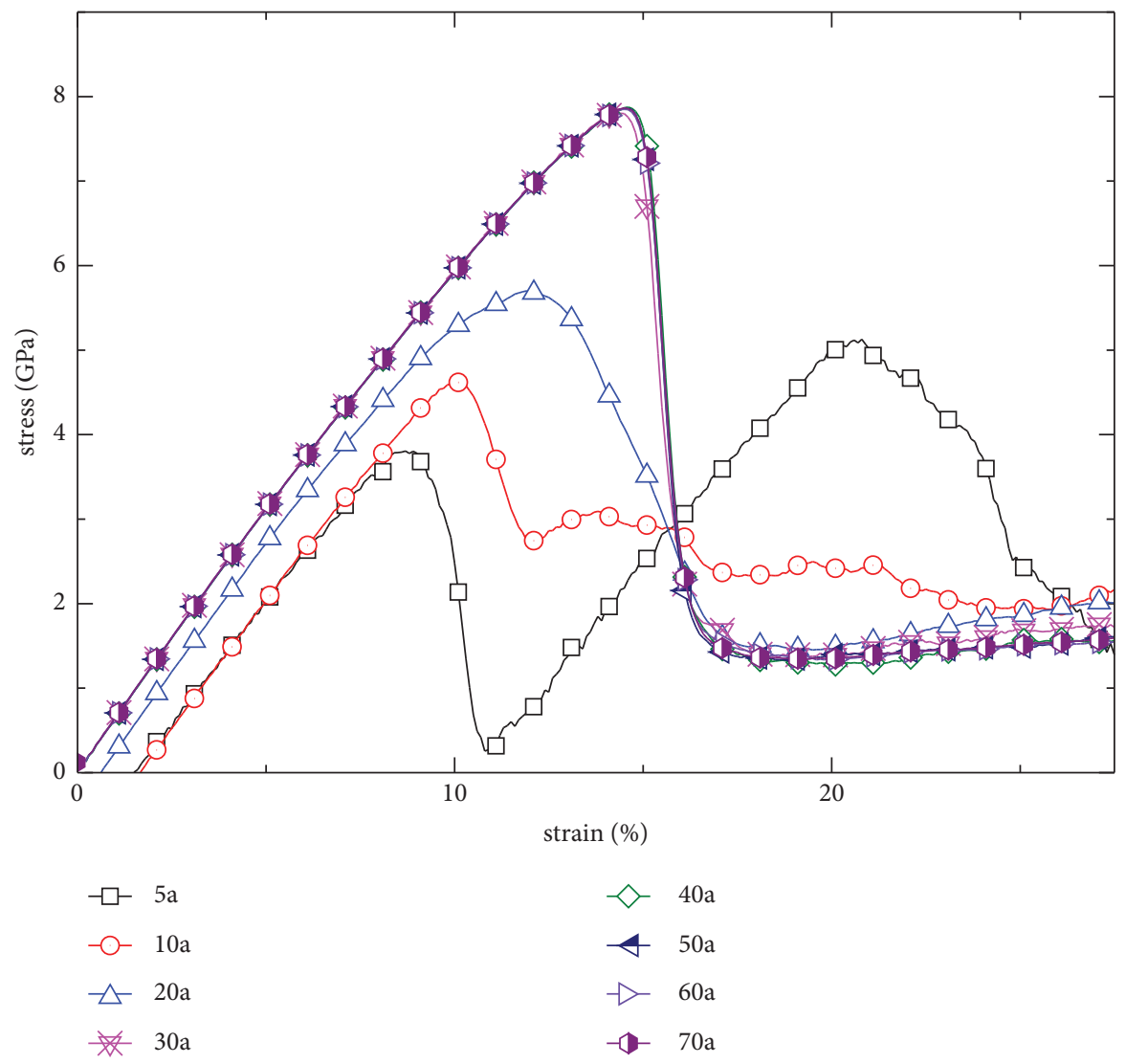

Figure 1: The stress-strain curves of boxes with different sizes such as $5 a, 10 a, \ldots 70 a$, where $a=0.405 \mathrm{~nm}$.

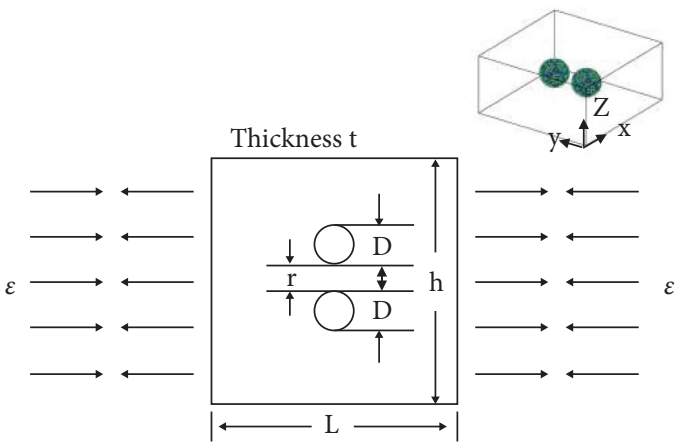

Figure 2: Loading conditions and dimensions designed for simulations.

unloaded, and after the equilibrium of $20 \mathrm{ps,} \mathrm{the} \mathrm{strain} \mathrm{rate}$ controlled tensile loading was performed. In order to better simulate the extreme loading conditions [8], the loading strain rate was set to $1 \times 10^{10} \mathrm{~s}^{-1}$. The specimens were visualized by the open visualization tool (OVITO) [30] (Figure 3), and all atoms were colored by the centrosymmetry parameter (CSP). The green lines in the visual image are the dislocations marked with CSP.

\section{Results and Discussion}

3.1. Comparison of the Void Coalescence under Uniaxial Compressive-Tensile Loading with Different Compressive
Strains. Figure 4 illustrates the stress-strain curves under different compressive strains, in which the curve could be divided into three stages. In the first stage, except for the compressive strains -7.5 and $-8 \%$, the stress varied linearly with respect to the strain at a similar rate. Meanwhile, it can be seen that during the compressive phase, the curves of the compressive strains -7.5 and $-8 \%$ were nonlinear, indicating that the material has entered the local plastic region. In the second stage, the stress-strain curve reached its peak, implying that the material yields. The enlarged section of Figure 4 shows that the yield strength fluctuates within a certain range before the initial compressive strain of $-7 \%$. From the compressive strain of $-7 \%$, the yield strength decreases significantly. However, when the compressive strain is $-8 \%$, the yield strength is higher than when the compressive strain is $-7.5 \%$. In addition, as the initial compressive strain decreases, the material will yield in advance. The curves enter the platform section in the third stage, which also represents that the material enters the plastic flow section.

To analyze the phenomena appearing in the stress-strain curves more clearly, the model was visualized by OVITO, taking the center of the two voids as the $Y$-axis and slicing in the $X$-direction to obtain the profile at different moments. This is equivalent to selecting a length on the $Z$-axis to study the plane strain issue. Hence, there is no need to include the influence of the boundary conditions in the $Z$-direction on the simulation. Three important moments have been 


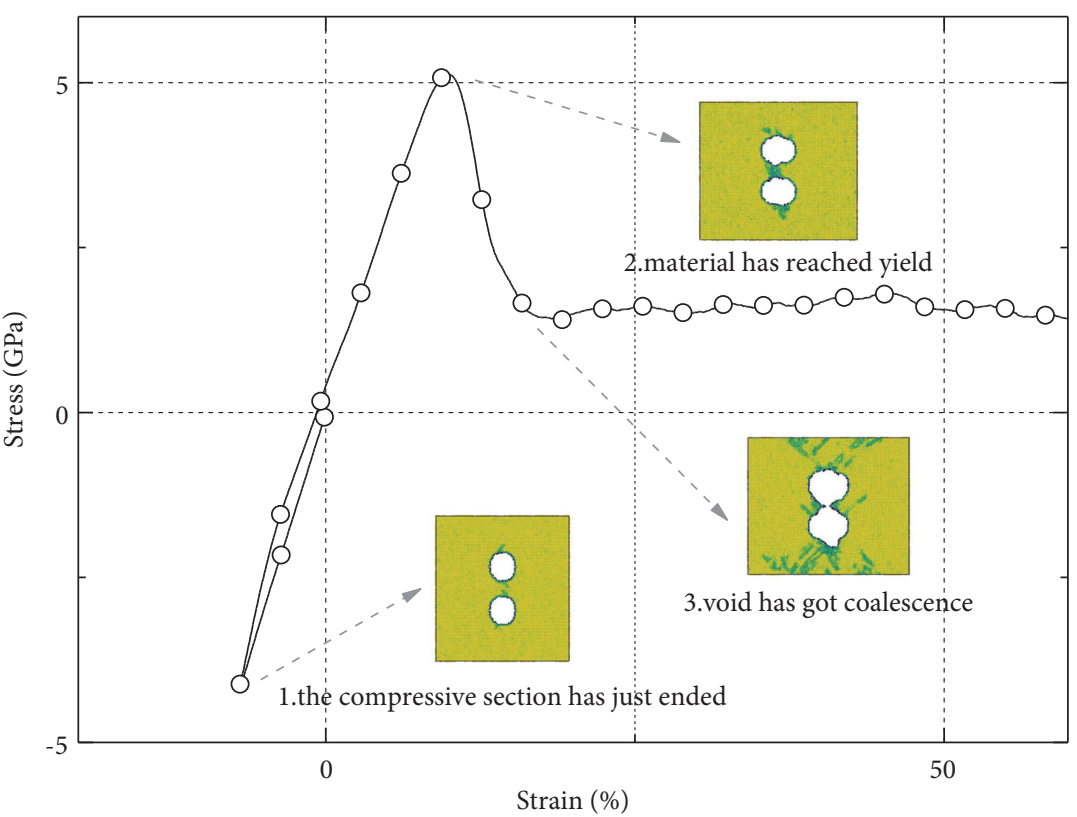

(a)
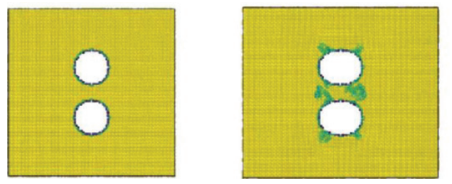

(c)
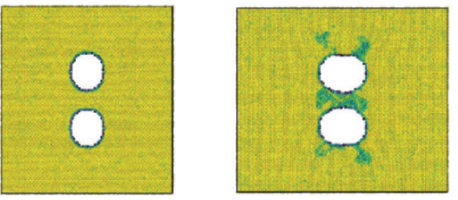

(e)

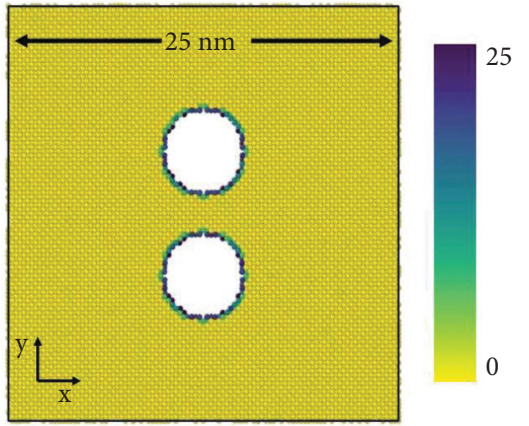

(b)
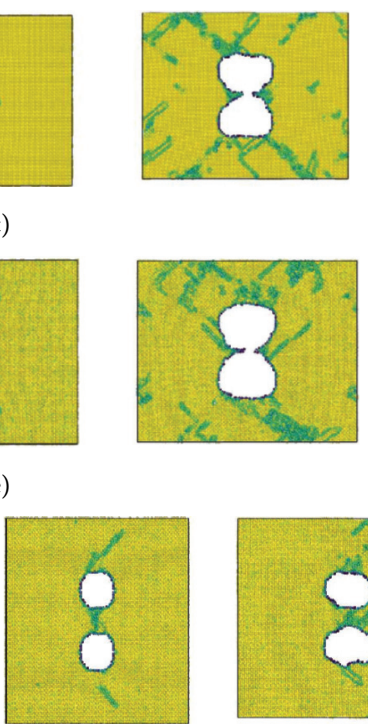

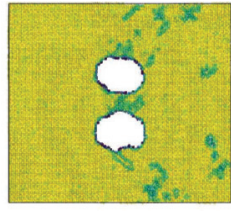

(g)
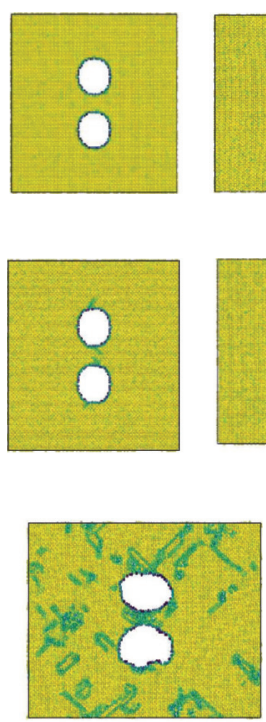

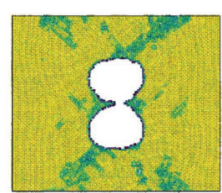

(d)
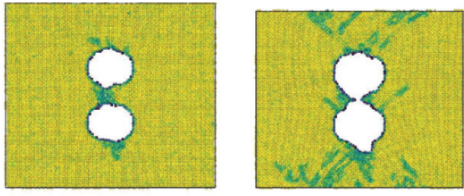

(f)

Figure 3: (a) Three moments as the analysis objectives; (b) the snapshot of section view; (c-f) the atomic snapshot of different compressive strains at the "three important moments" shown in Figure 3(a). (a) Three important moments: 1. the compressive section has just ended; 2. the material has reached yield; 3 . the void has coalescence. (c) $\varepsilon_{i c}=0 \%$. (d) $\varepsilon_{i c}=-5 \%$. (e) $\varepsilon_{i c}=-6 \%$. (f) $\varepsilon_{i c}=-7 \%$. (g) $\varepsilon_{i c}=-8 \%$.

selected as the objectives of the analysis in $-7 \%$ of the compressive strain sample ( Figure 3(a)). Three moments correspond to the snapshots in Figures 3(b) $-3(\mathrm{~g})$, which are "the compressive phase has just ended," "the material has reached its yield $(\varepsilon \approx 0.1)$," and "the void has got coalescence $(\varepsilon \approx 0.2)$." Figure $3(b)$ is the section view of the initial configuration, where the axis and size are marked in Figure 3(b). Figure 3(c) shows that the voids are continuously developing. After growing to a certain size under uniaxial tensile loading, the ligament that is at the center of two voids begins necking and then the voids coalesce. In the second image, dislocations are generated between two voids.
After coalescence, the dislocations originating from the voids continue to propagate. Note that Figures $3(\mathrm{~d})-3(\mathrm{~g})$ show that the voids still coalesce. In addition, when the initial compressive strain is $-5 \%$, there are no obvious dislocations after the compressive phase. In the second section, symmetric dislocations are also generated between two voids and in the process of void coalescence. Similarly, dislocations form an X-shaped distribution [31]. A different phenomenon can be observed where dislocations are generated between the voids at the end of the compression when the compressive strain is greater than $-7 \%$, and then dislocations evolve into an asymmetric distribution. The result 


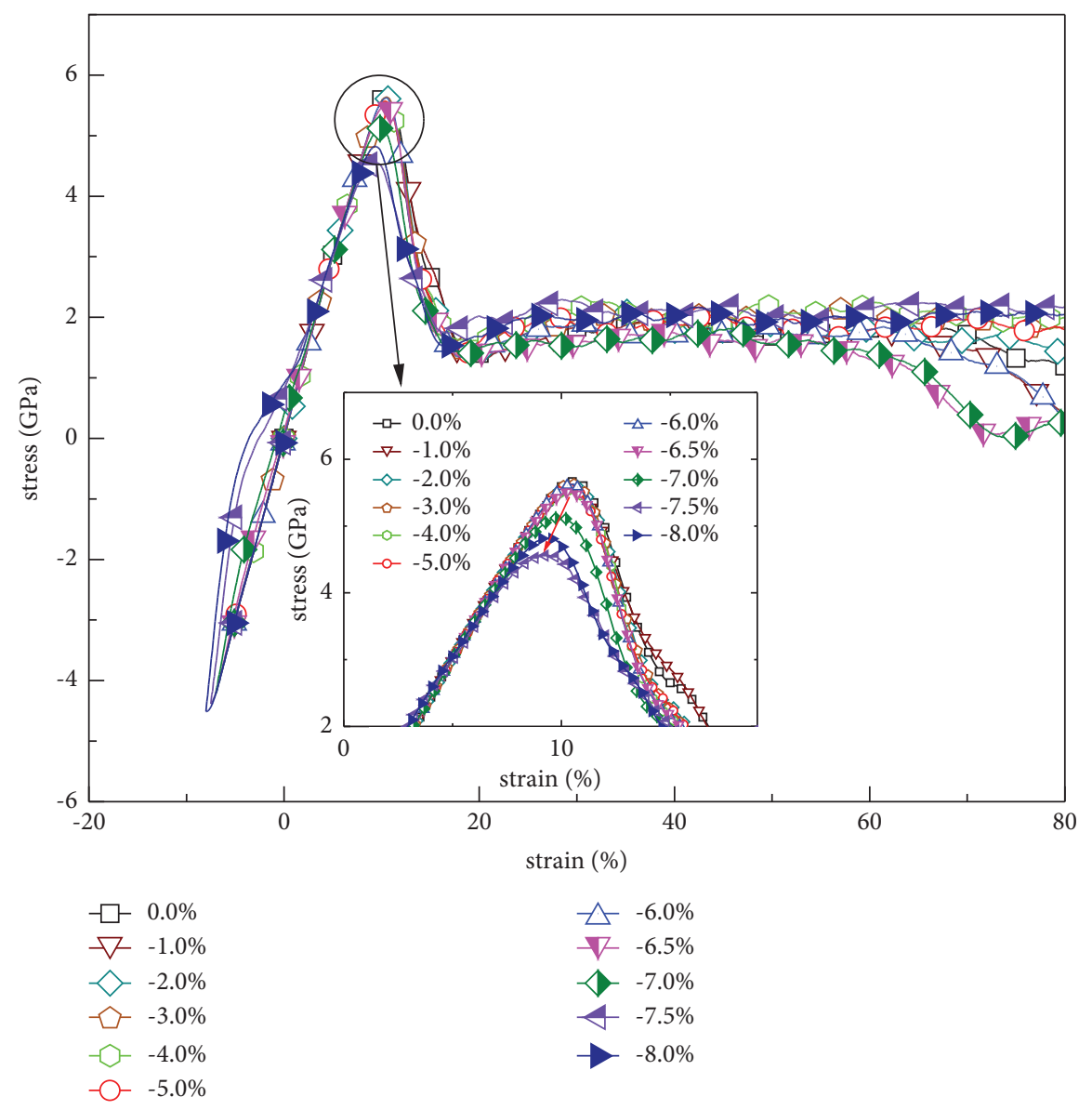

FIGURE 4: Stress-strain curves of different compressive strains (the inset is a partial enlarged view of the yield strength in the stress-strain curve).

shows that although the voids ultimately coalescence, dislocations have already occurred around the voids when the compressive phase has ended, as shown in Figure 3(e). Figure $3(\mathrm{~g})$ shows that the voids also generate dislocations at the end of the compressive phase, but no coalescence occurs. In addition, dislocations are generated between the voids and are emitted in an approximate direction of $43^{\circ}$ from the tensile direction under the compressive phase.

In MD simulation, the void coalescence is obtained based on statistical methods, which is generally determined by merging two voids shown in the atomic snapshot. However, so far, there are no suitable parameters to depict the void coalescence, so we apply the coalescence strain to describe the void coalescence; that is, when two voids merge in the atomic snapshot, the current strain is recorded and defined as the coalescence strain. When the strain is equal to the coalescence strain, the voids coalesce. Figure 5 shows the relationship between the initial compressive strain and coalescence strain. As the initial compressive strain increases, the coalescence strain also increases, and both increase linearly before the initial compressive strain of $-7 \%$. When the initial strain is $-7.5 \%$, because dislocations have already occurred during the compressive phase, the void coalescence begins to become more difficult. Figure 6(a) illustrates the relationship between the yield strength and

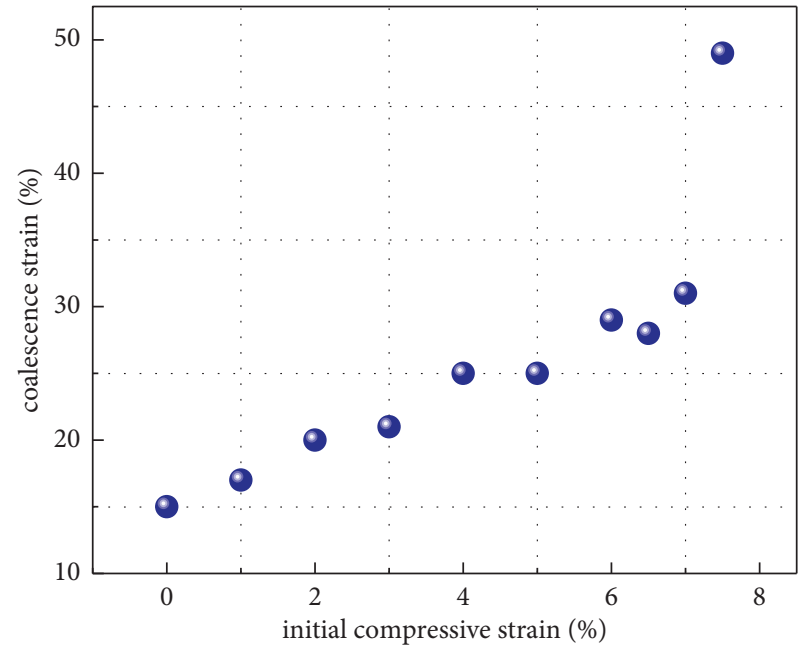

FIgURE 5: Relationship between the initial compressive strain and coalescence strain.

compressive strain. It can be clearly observed from the figure that before the compressive strain of $-7 \%$, the yield strength fluctuates around 5.6 GPa (blue dash line). After the compressive strain of $-7 \%$, there is a significant decrease in the 


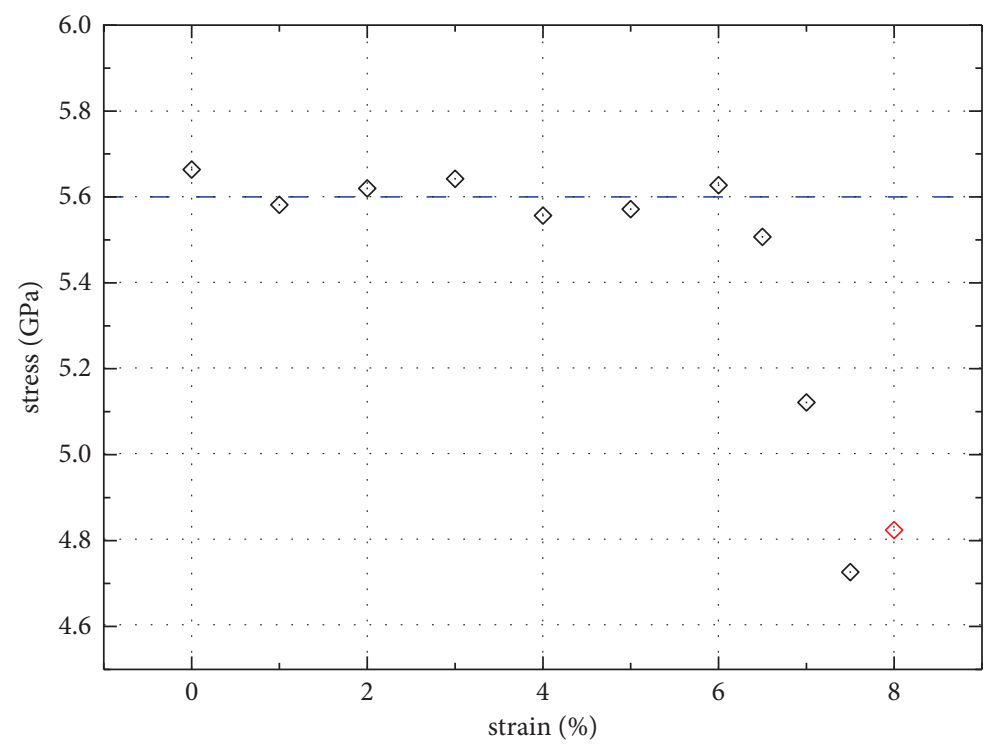

$\diamond$ Coalescence

$\diamond$ Not coalescence

(a)

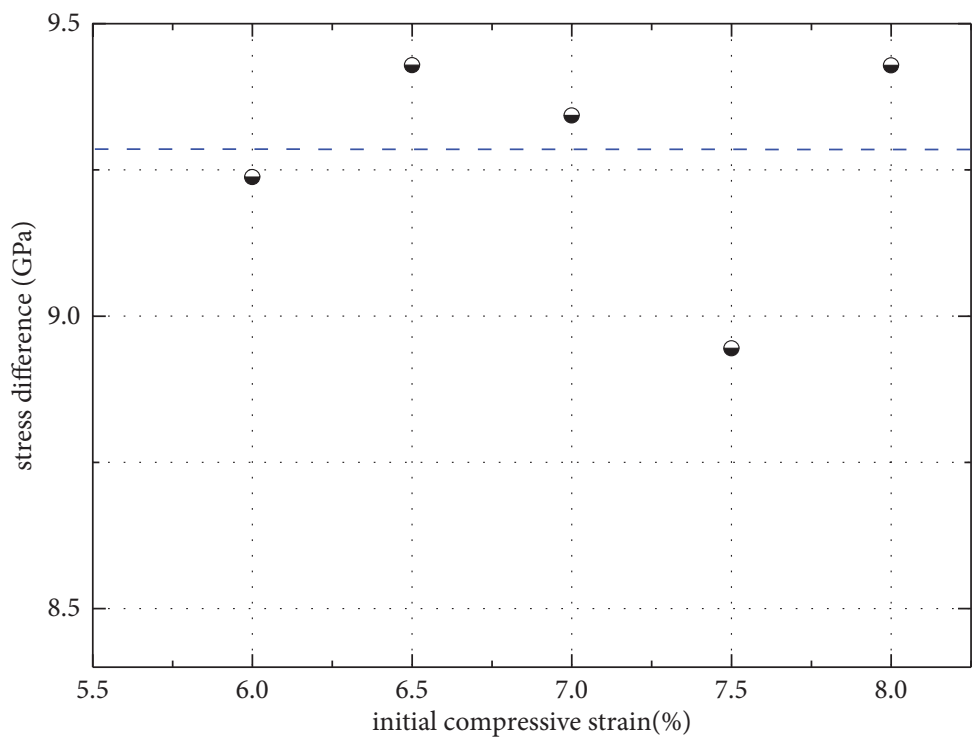

(b)

Figure 6: (a) Initial compressive strain vs. yield stress; (b) the relationship between the stress difference and compressive strain.

yield stress, and when the compressive strain is $-8 \%$, the yield strength is higher than when the compressive strain is $-7.5 \%$. The decline in the yield strength is due to the existence of BE. For further clarification, Figure 6(b) illustrates the relationship between the stress difference and compressive strain. Except for the sample with a compressive strain of $-6 \%$, all critical stress differences and the minimum stress difference fluctuate in the vicinity of the blue dash line, which is similar to what is described in the BE [32]. In addition, Figure 6(a) shows that the yield strength with a compressive strain of $-8 \%$ is higher than that with a compressive strain of $-7.5 \%$, while for the compressive strain of $-7.5 \%$, the voids do not coalescence. This phenomenon why the voids cannot coalescence will be explained in next section.

3.2. Dislocation Analysis of the Void Coalescence under Compressive-Tensile Loading. In order to profoundly understand the effect of the compressive phase on the void coalescence, Figures 7 and 8 show the dislocation processing obtained by different methods at the end of the compressive phase. In Figure 7, CSP is employed to depict the dislocation, in which Figures 7 (a)-7(c) illustrate that the specimen with an initial compressive strain from -5 to $-6.5 \%$ has few dislocations during the compressive phase. Obviously, 


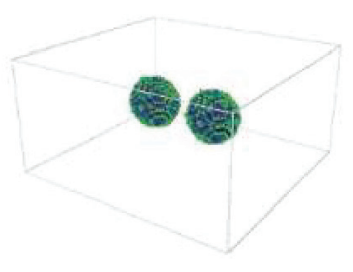

(a)

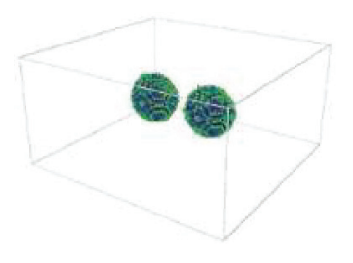

(b)

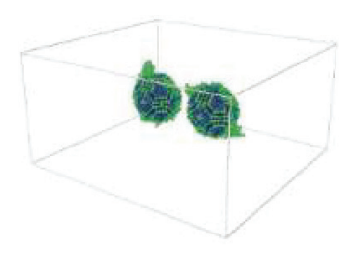

(c)

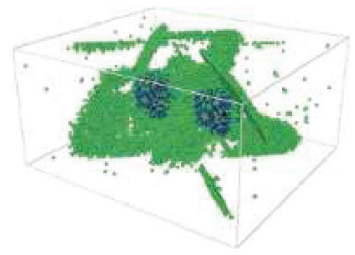

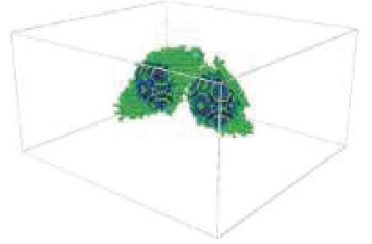

(d)

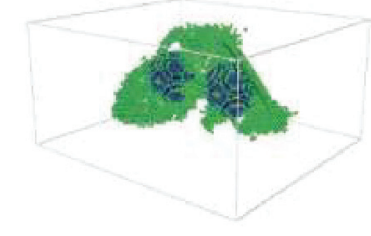

(e)

(f)

FIgURE 7: Dislocation emission by the void surface by CSP. (a) $\varepsilon_{i c}=-5 \%$. (b) $\varepsilon_{i c}=-6 \%$. (c) $\varepsilon_{i c}=-6.5 \%$. (d) $\varepsilon_{i c}=-7 \%$. (e) $\varepsilon_{i c}=-7.5 \%$. (f) $\varepsilon_{i c}=-8 \%$.

dislocations are generated in the specimen when the compression strains are -7 and $-8 \%$ (Figures $6(\mathrm{~d})-6(\mathrm{f})$ ), so that an increase of the initial compressive strain would cause the dislocation to increase. A dislocation begins to form, mainly on the void surface, which connects into a piece in the middle of the voids and finally expands to the material boundary. Moreover, it is not isotropic when it expands, and the voids are arranged in more dislocations. Figure 8 shows the dislocation lines that are extracted by the dislocation extraction algorithm (DXA) $[30,33]$. The dislocation density $\rho_{d}$ can be calculated by the length of the dislocation line:

$$
\rho_{d}=\frac{l}{V}
$$

where $V$ is the total volume of the box at the initial moment, and $l$ is the length of the dislocation line. As shown in Figure 8 , in the compressive phase, the dislocations are produced continuously, which results in an increase in the dislocation density that affects the void coalescence. From the surface of two spherical voids, dislocations are absorbed in the form of dislocation lines which are formed around the voids. As the strain increases, both dislocation lines merge into a large dislocation group, which is accompanied by lots of disorderly dislocation bands. In the compressive phase, the evolution of voids is also accompanied by the adsorption-mediated growth of dislocations, similar to those found by Sills [34]. Figure 8(c) shows that after applying $-8 \%$ compressive strain, the dislocations grow in a large amount, and the growth has an effect on the later tensile phase. Therefore, the dislocation density with $-8 \%$ compressive strain is compared with $-5 \%$ compressive strain. Figure 9 shows the dislocation density-stress-time curve of the voids, in which it can be seen that both samples in the first 5 ps are under compressive stress, so that the $0-5$ ps segment is coincident. In the 5-8 ps stage, dislocations begin to grow quickly, and the dislocation density increases rapidly in the sample, for which the compressive strain is $-8 \%$. The dislocation density begins to decrease during $8-20$ ps because the tensile stress begins to dominate the dislocation movement. It should be noted that the stress is nonlinear, which indicates that the voids have formed a local plastic zone at this time in the stress-time diagram. At the $20-30$ ps stage, dislocations begin to form in the specimen with a compressive strain of $-5 \%$, and the time 20 ps corresponds to the moment when stress reaches the yield. Compared to the compressive strain of $-5 \%$, the dislocation of the sample with a compressive strain of $-8 \%$ grows in the compressive phase, and it causes the yield point hysteresis on the stresstime curve. After $30 \mathrm{ps}$, both the stress-time and densitytime curves enter the platform stage.

The beginning of plastic deformation is the slip of dislocations, and the large-scale evolution of dislocations makes other internal dislocations difficult to move. The direction of dislocation movement depends on the BE. Due to the presetting compressive strain, dislocations are generated in the void surface which causes back stress in the material. The pilling up of dislocations leads to the dislocations where closed ligaments in the tensile phase cannot continue to slip. In addition, the back stress prevents the positive direction deformation, which results in a decrease in the yield strength while the voids do not coalesce.

\subsection{Temperature Effect under Compressive Loading on the} Void Coalescence. The effects of temperature and strain rate on the void coalescence should also be considered in studying extreme conditions. Figure 10 is a snapshot of the specimen under different temperatures subjected to compressive-tensile loading, in which the compressive strain is set to $-5 \%$. As can be seen, the effect of temperature on the void coalescence is obvious. For instance, when a strain is $20 \%$, the voids in the 300 and $500 \mathrm{k}$ get coalescence, while the void coalescence in the $100 \mathrm{k}$ sample occurs at a strain of 50\%, as shown in Figure 10(c). This behavior implies that a decrease in temperature will suppress the void coalescence. In addition, as the temperature increases, the yield strength gradually decreases. Therefore, the low temperature would inhibit the void coalescence. From the energy perspective, 


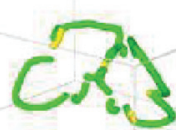

(a)

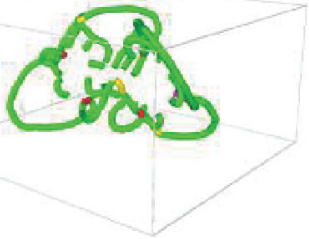

(b)

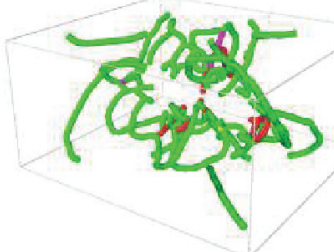

(c)

FiguRE 8: The dislocation lines for the compressive strain from the void surface $(-7 \%-8 \%)$. (a) $\varepsilon_{i c}=-7 \%$, (b) $\varepsilon_{i c}=-7.5 \%$, and (c) $\varepsilon_{i c}=-8 \%$.

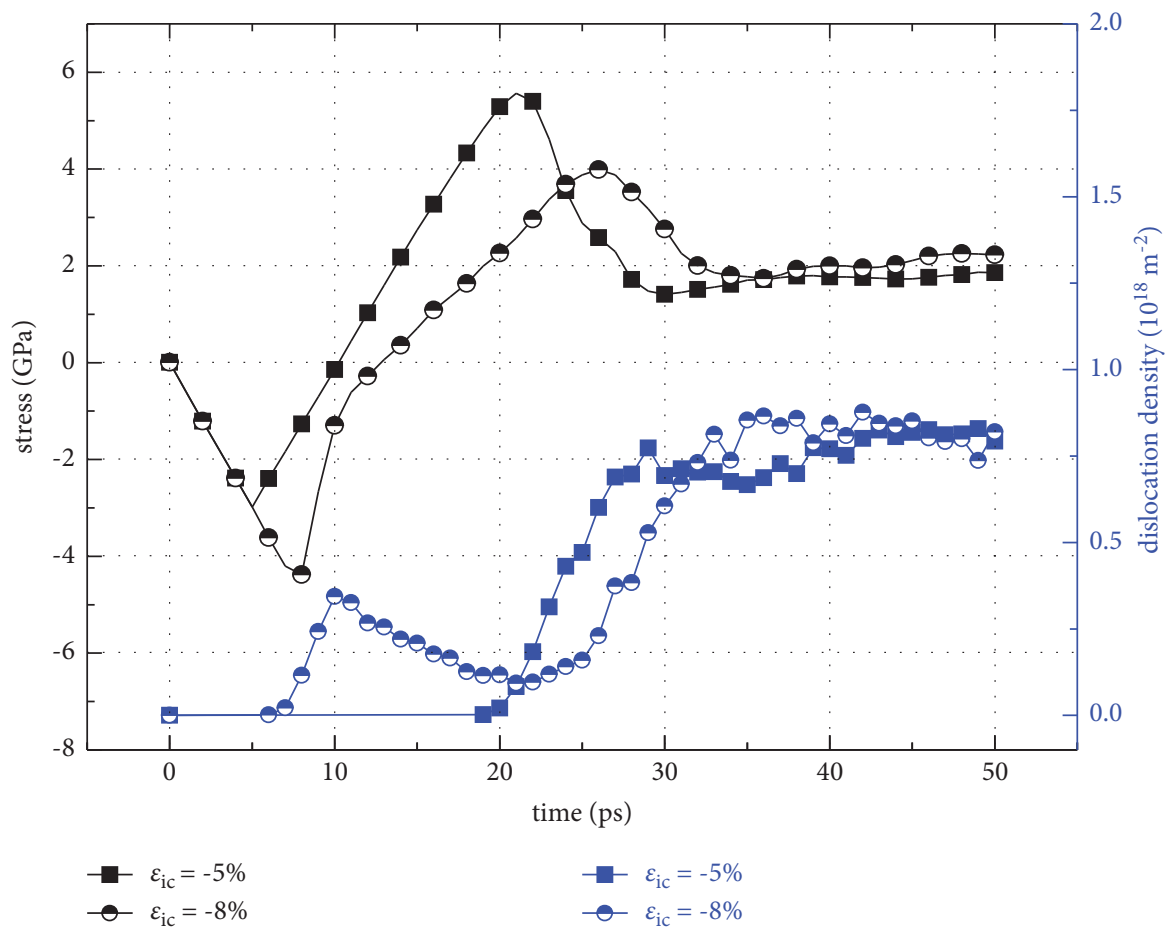

FIgURE 9: The curve of dislocation-stress-time with different compressive strains.

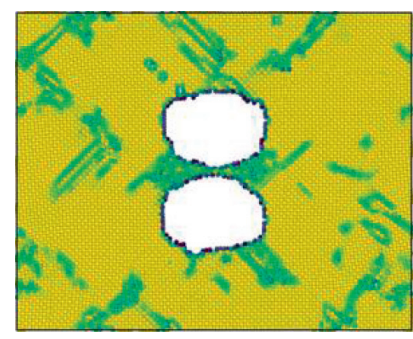

(a)

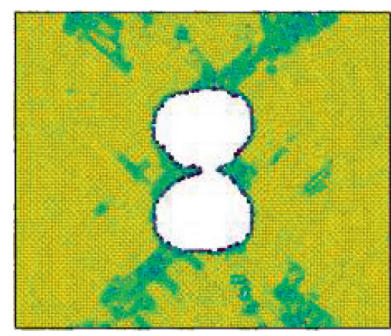

(b)

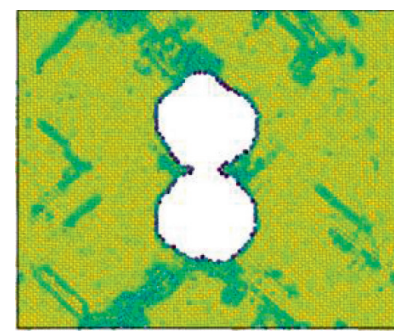

(c)

Figure 10: Continued. 


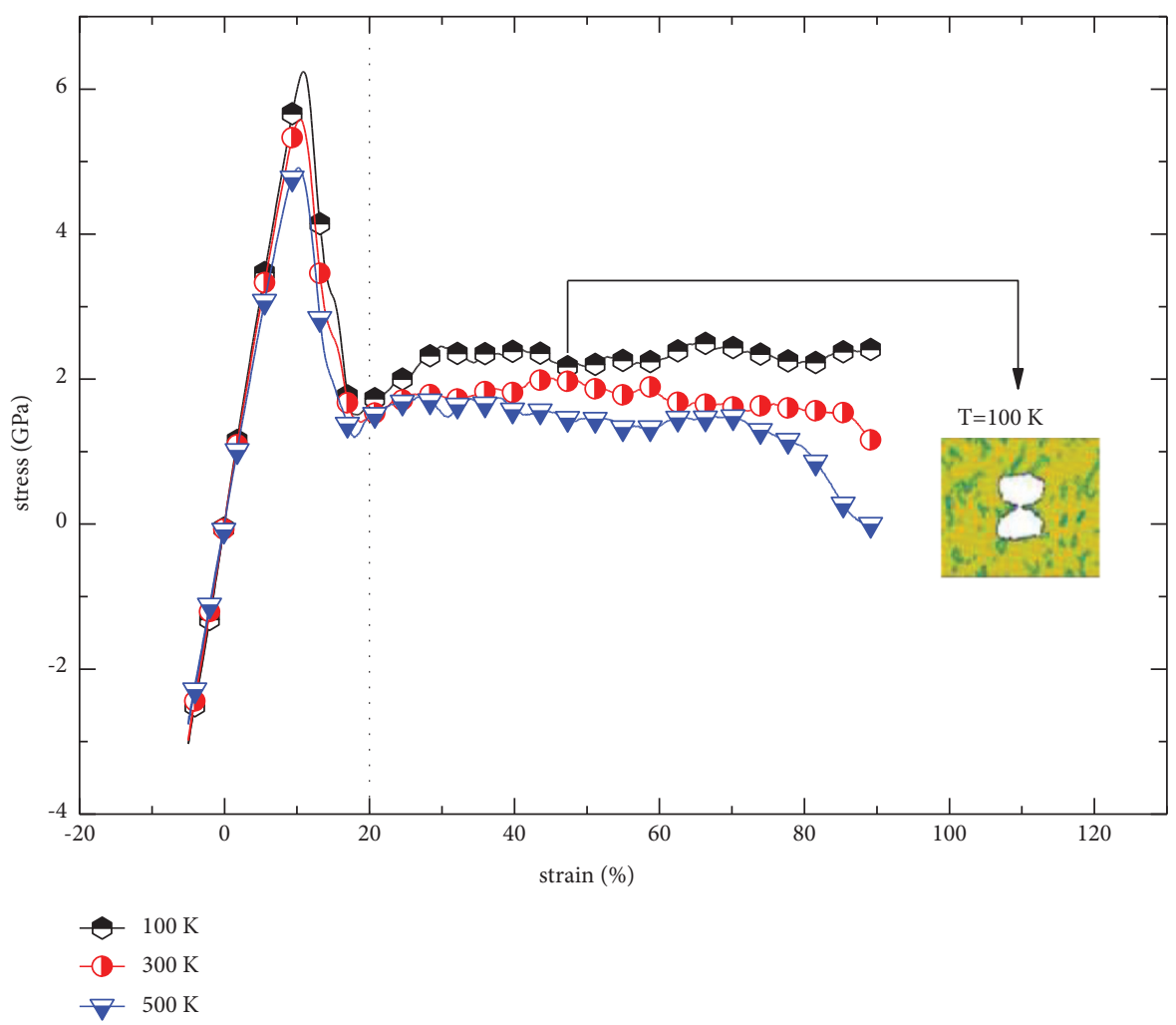

(d)

Figure 10: (a-c) The snapshots of the specimen with a strain of 20\%; (a) $100 \mathrm{k}$ at $\varepsilon=20 \%$. (b) $300 \mathrm{k}$ at $\varepsilon=20 \%$. (c) $500 \mathrm{k}$ at $\varepsilon=20 \%$. (d) The stress-strain curve at different temperatures.

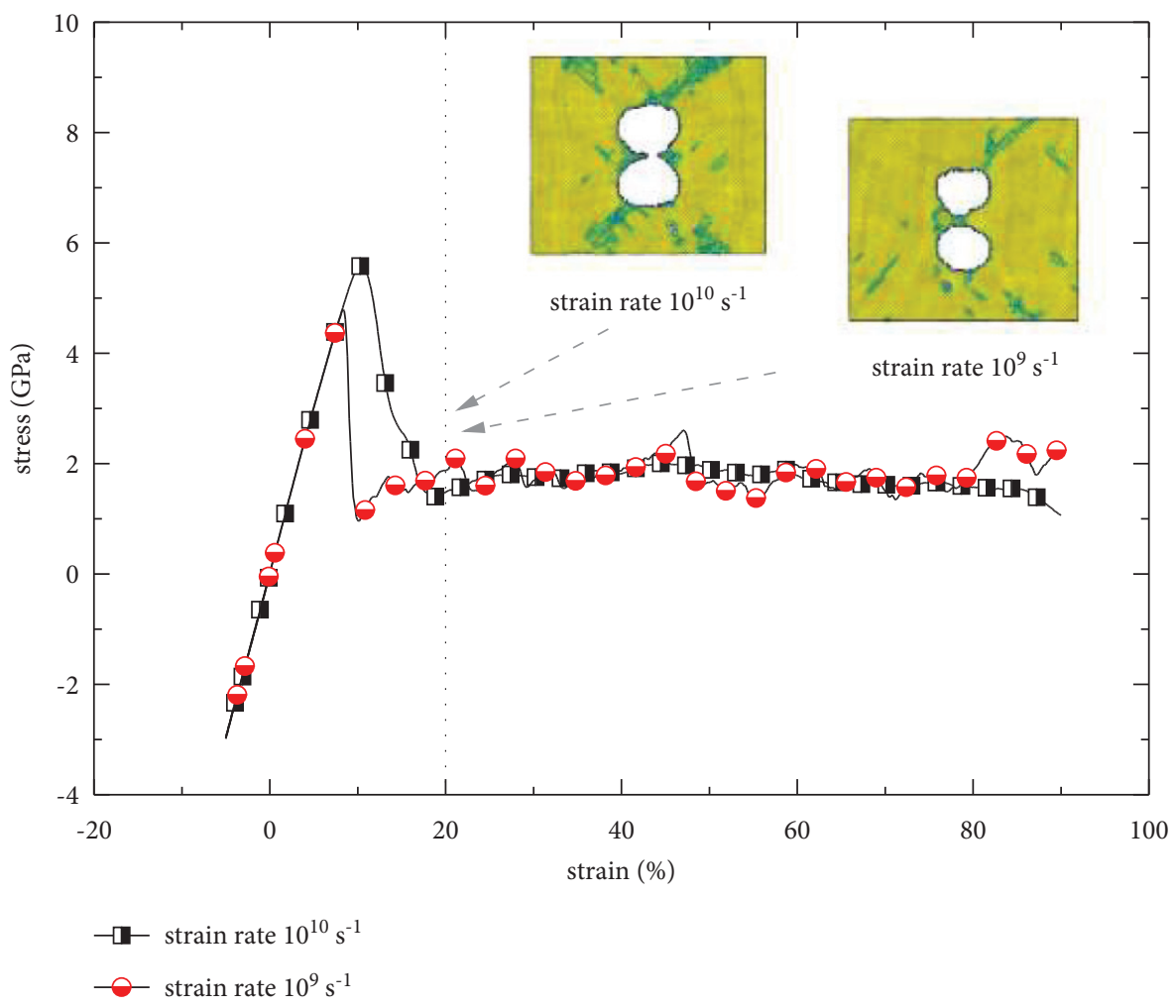

FIgURE 11: The stress-strain curve at different strain rates when the compressive strain is set to $-5 \%$. 
the transition and rearrangement of atoms can only obtain energy through external deformation, which makes it difficult for voids to coalesce. Similarly, the strain rate also has an influence on the void coalescence (Figure 11). After compressive and tensile loading, a decrease of the strain rate would cause more obvious noncoalescence of the voids. When the strain is $20 \%$, only the specimen whose strain rate is $10^{10} \mathrm{~s}^{-1}$ has coalescence. This shows that at a lower strain rate, the compression effect has a stronger influence on the voids, and it is necessary to consider the influence of compression on the void coalescence.

\section{Conclusions}

To understand why the void coalescence stopped after applying some compressive loading, the MD method was used in this paper to simulate single crystal aluminum under uniaxial compressive-tensile plastic deformation with nanovoids. Moreover, the effects of temperature and strain rate on the void coalescence were investigated, and the yield strength of specimens under different compressive strains was analyzed. It was found that the compression not only reduces the yield strength of the material, but also obstructs the void coalescence, which also modifies the existing damage constitutive model. The main conclusions are as follows:

(i) Before the compressive strain of $-7 \%$, the yield strength fluctuates within a certain range, and the yield strength decreases with the increasing compressive strain after - $7 \%$. Compared with the uniaxial tensile loading, this behavior can be explained by BE. Note that if the compression effect is not considered in engineering applications and material manufacturing, it will cause damage to the material before its service life, posing a great risk.

(ii) The compressive phase causes dislocation absorption at the void ligament, and the dislocation growth obstructs the dislocation movement during the tensile phase. Therefore, the compressive phase obstructs the void coalescence. This is why the yield strength of $\varepsilon_{i c}=-8 \%$ is higher than $\varepsilon_{i c}=-7.5 \%$. The temperature and strain rate also have an obvious effect on the void coalescence under compressive and tensile loading. A low temperature would suppress the void coalescence, and the strain rate would inhibit the void coalescence as well.

\section{Data Availability}

The data used to support the findings of this study are available from the corresponding author upon request.

\section{Conflicts of Interest}

The authors declare that they have no conflicts of interest.

\section{Authors' Contributions}

Yongmao Pei conceptualized the study; Wei Ning curated the data; Ruichen Tu was responsible for formal analysis;
Ruichen Tu investigated the study; Yan Liu developed the methodology of the study; Fengguo Zhang was responsible for project administration; Ruichen Tu and Wei Ning were responsible for software; Yongmao Pei and Dongsheng Zhang were responsible for supervision; Ruichen Tu and Yan Liu contributed to the visualization of the study; Ruichen Tu wrote the original draft; Wei Ning and Yongmao Pei reviewed and edited the manuscript.

\section{Acknowledgments}

This work was supported by the Science Challenge Project (No. TZ2018001), the Shaanxi Provincial Science and Technology Department (No. 2016JM5039), and the Science and Technology Project of Shaanxi University (No. SLGQD14-03).

\section{References}

[1] V. Tvergaard, "Influence of voids on shear band instabilities under plane strain conditions," International Journal of Fracture, vol. 17, no. 4, pp. 389-407, 1981.

[2] V. Tvergaard, "On localization in ductile materials containing spherical voids," International Journal of Fracture, vol. 18, no. 4, pp. 237-252, 1982.

[3] V. Tvergaard and A. Needleman, "Analysis of the cup-cone fracture in a round tensile bar," Acta Metallurgica, vol. 32, no. 1, pp. 157-169, 1984.

[4] A. L. Gurson, "Continuum theory of ductile rupture by void nucleation and growth: Part I-yield criteria and flow rules for porous ductile media," Journal of Engineering Materials and Technology, vol. 99, pp. 2-15, 1997.

[5] M. Gologanu, J.-B. Leblond, and J. Devaux, "Approximate models for ductile metals containing non-spherical voidsCase of axisymmetric prolate ellipsoidal cavities," Journal of the Mechanics and Physics of Solids, vol. 41, no. 11, pp. 1723-1754, 1993.

[6] M. Gologanu, J.-B. Leblond, and J. Devaux, "Approximate models for ductile metals containing $\mathrm{n}$ voids-case of axisymmetric oblate ellipsoidal cavities," Journal of Engineering Materials and Technology, vol. 116, no. 3, pp. 290-297, 1994.

[7] B. Nicola, R. Andrew, S. Martin, and N. Michael, "Investigation on flying plate diameter to thickness ratio influence on damage pattern and spall signal," International Journal of Impact Engineering, vol. 29, no. 1-10, pp. 127-138, 2003.

[8] J. P. Cuq-Lelandais, M. Boustie, L. Berthe et al., "Spallation generated by femtosecond laser driven shocks in thin metallic targets," Journal of Physics D Applied Physics, vol. 42, no. 6, Article ID 065402, 2009.

[9] P. F. Thomason, "Ductile spallation fracture and the mechanics of void growth and coalescence under shock-loading conditions," Acta Materialia, vol. 47, no. 13, pp. 3633-3646, 1999.

[10] G. I. Kanel, S. V. Razorenov, K. Baumung, and J. Singer, "Dynamic yield and tensile strength of aluminum single crystals at temperatures up to the melting point," Journal of Applied Physics, vol. 90, no. 1, pp. 136-143, 2001.

[11] Z.-P. Wang, "Void-containing nonlinear materials subject to high-rate loading," Journal of Applied Physics, vol. 81, no. 11, pp. 7213-7227, 1997.

[12] V. Tvergaard, "Effect of void size difference on growth and cavitation instabilities," Journal of the Mechanics and Physics of Solids, vol. 44, no. 8, pp. 1237-1253, 1996. 
[13] E. T. Seppälä, J. Belak, and R. E. Rudd, "The onset of void coalescence during dynamic fracture of ductile metals," Physical Review Letters, vol. 93, no. 24, Article ID 245503, 2005.

[14] N. Jacques, S. Mercier, and A. Molinari, "Effects of microscale inertia on dynamic ductile crack growth," Journal of the Mechanics and Physics of Solids, vol. 60, no. 4, pp. 665-690, 2012.

[15] A. Molinari, S. Mercier, and N. Jacques, "Dynamic failure of ductile materials," Procedia Iutam, vol. 10, pp. 201-220, 2014.

[16] V. A. Lubarda, M. S. Schneider, D. H. Kalantar, B. A. Remington, and M. A. Meyers, "Void growth by dislocation emission," Acta Materialia, vol. 52, no. 6, pp. 1397-1408, 2004.

[17] M. F. Horstemeyer, M. M. Matalanis, A. M. Sieber, and M. L. Botos, "Micromechanical finite element calculations of temperature and void configuration effects on void growth and coalescence," International Journal of Plasticity, vol. 16, no. 7, pp. 979-1015, 2000.

[18] G. P. Potirniche, M. F. Horstemeyer, G. J. Wagner, and P. M. Gullett, "A molecular dynamics study of void growth and coalescence in single crystal nickel," International Journal of Plasticity, vol. 22, no. 2, pp. 257-278, 2006.

[19] T. Tang, S. Kim, and M. F. Horstemeyer, "Molecular dynamics simulations of void growth and coalescence in single crystal magnesium," Acta Materialia, vol. 58, no. 14, pp. 4742-4759, 2010.

[20] Y. Cui and Z. Chen, "Molecular dynamics simulation of the influence of elliptical void interaction on the tensile behavior of aluminum," Computational Materials Science, vol. 108, pp. 103-113, 2015.

[21] X. Deng, W. Zhu, Y. Zhang, H. He, and F. Jing, "Configuration effect on coalescence of voids in single-crystal copper under shock loading," Computational Materials Science, vol. 50, no. 1, pp. 234-238, 2011.

[22] Y. Zhang and S. Jiang, "Investigation on dislocation-based mechanisms of void growth and coalescence in single crystal and nanotwinned nickels by molecular dynamics simulation," Philosophical Magazine, vol. 97, no. 30, pp. 1-23, 2017.

[23] X. Peng, W. Zhu, K. Chen, X. Deng, and Y. Wei, "Molecular dynamics simulations of void coalescence in monocrystalline copper under loading and unloading," Journal of Applied Physics, vol. 119, no. 16, Article ID 165901, 2016.

[24] M. J. Diwu and X. M. Hu, "Plastic deformation in nanoporous aluminum subjected to high-rate uniaxial compression," Acta Physica Sinica, vol. 63, Article ID 170201, 2015.

[25] L. Y. Zhao and Y. Liu, "Investigation on void growth and coalescence in single crystal copper under high-strain-rate tensile loading by atomistic simulation," Mechanics of Materials, vol. 151, Article ID 103615, 2020.

[26] S. Plimpton, "Fast parallel algorithms for short-range molecular dynamics," Journal of Computational Physics, vol. 117, no. 1, pp. 1-19, 1995.

[27] M. S. Daw and M. I. Baskes, "Embedded-atom method: derivation and application to impurities, surfaces, and other defects in metals," Physical Review B, vol. 29, no. 12, pp. 6443-6453, 1984.

[28] Y. Mishin, D. Farkas, M. J. Mehl, and D. A. Papaconstantopoulos, "Interatomic potentials for monoatomic metals from experimental data andab initiocalculations," Physical Review B, vol. 59, no. 5, pp. 3393-3407, 1999.

[29] W. Shinoda, M. Shiga, and M. Mikami, "Rapid estimation of elastic constants by molecular dynamics simulation under constant stress," Physical Review B, vol. 69, no. 13, Article ID 134103, 2004.

[30] A. Stukowski, "Visualization and analysis of atomistic simulation data with OVITO-the Open Visualization Tool," Modelling and Simulation in Materials Science and Engineering, vol. 18, no. 1, pp. 2154-2162, 2010.

[31] C. L. Kelchner, S. J. Plimpton, and J. C. Hamilton, "Dislocation nucleation and defect structure during surface indentation," Physical Review B, vol. 58, no. 17, pp. 11085-11088, 1998.

[32] A. Thakur, S. Nemat-Nasser, and K. S. Vecchio, "Dynamic bauschinger effect," Acta Materialia, vol. 44, no. 7, pp. 2797-2807, 1996.

[33] L. A. Zepeda-Ruiz, A. Stukowski, T. Oppelstrup, and V. V. Bulatov, "Probing the limits of metal plasticity with molecular dynamics simulations," Nature, vol. 550, no. 7677, pp. 492-495, 2017.

[34] R. B. Sills and B. L. Boyce, "Void growth by dislocation adsorption," Materials Research Letters, vol. 8, no. 3, pp. 103-109, 2020. 